\title{
Tumor-Infiltrating Lymphocytes in Biopsy Specimens Obtained 7 Days after Starting Chemoradiotherapy for Rectal Cancer Are Predictors of the Response to Chemoradiotherapy
}

\author{
Hiroshi Miyakita $^{\text {a }}$ Sotaro Sadahiro $^{\text {a }}$ Toshiyuki Suzuki $^{a}$ Lin Fung Chan ${ }^{\text {a }}$ \\ Takashi Ogimi $^{a}$ Kazutake Okada ${ }^{a}$ Seiichiro Yamamoto ${ }^{a}$ Hiroshi Kajiwara ${ }^{b}$ \\ aDepartment of Surgery, Tokai University School of Medicine, Isehara, Japan; 'bepartment of Pathology, \\ Tokai University School of Medicine, Isehara, Japan
}

\section{Keywords}

Tissue-infiltrating lymphocytes · Rectal cancer ·

Chemoradiotherapy

\begin{abstract}
Background: Neoadjuvant chemoradiotherapy (nCRT) followed by total mesorectal excision surgery is a standard treatment for locally advanced rectal cancer (LARC). Tumorinfiltrating lymphocytes (TILs) have been reported to be associated with tumor response; however, this remains to be established. We previously reported that histological changes on biopsy specimens obtained 7 days after starting nCRT are strong predictors of response to nCRT. Methods: The subjects were 208 patients with LARC who received nCRT. TILs on hematoxylin-eosin staining together with immunohistochemical staining of lymphocyte surface markers including CD3, CD4, CD8, and FoxP3 were performed both on the biopsy specimens before and 7 days after starting $\mathrm{nCRT}$. Results: The proportions of patients with high densities of $\mathrm{CD} 3+, \mathrm{CD} 4+, \mathrm{CD} 8+$, and FoxP3+ cells 7 days after starting CRT were significantly lower than the respective values before starting nCRT $(p<0.0001, p<0.0001, p=0.0023$, and
\end{abstract}

karger@karger.com www.karger.com/ocl

Karger $\stackrel{\text { ' }}{5}$

GOPEN ACCESS
(C) 2020 The Author(s)

Published by S. Karger AG, Basel

This is an Open Access article licensed under the Creative Commons Attribution-NonCommercial-4.0 International License (CC BY-NC) (http://www.karger.com/Services/OpenAccessLicense), applicable to the online version of the article only. Usage and distribution for commercial purposes requires written permission. $p=0.0046)$. In biopsy specimens obtained before treatment, high-density CD4+ cells and FOXP3+ cells were significantly associated with tumor shrinkage rate. High-density FOXP3+ cells were significantly associated with marked tumor regression. In biopsy specimens obtained 7 days after starting treatment, high-density CD4+ cells were significantly associated with marked tumor regression, tumor regression grade 1, and tumor shrinkage rate. High-density FoxP3+ cells were significantly associated with marked tumor regression and tumor shrinkage rate. Conclusions: In patients who received $\mathrm{nCRT}$ for LARC, the evaluations of immunohistochemical staining for CD4+ and FOXP3+ TILs were more intimately related to histological response to CRT and tumor shrinkage rates in biopsy specimens obtained 7 days after starting treatment than in biopsy specimens obtained before CRT.

(c) 2020 The Author(s)

Published by S. Karger AG, Basel

\section{Introduction}

Several large randomized controlled trials have demonstrated that multidisciplinary treatment significantly decreases the risk of local failure in patients with locally 
advanced rectal cancer $[1,2]$. Neoadjuvant chemoradiotherapy (nCRT) followed by total mesorectal excision is standard treatment for locally advanced rectal cancer.

The histological response to CRT has been reported to be closely related to oncologic outcomes $[3,4]$. We previously reported that histological changes on hematoxylineosin (H-E)-stained specimens together with immunohistochemical expression of p21 and apoptosis in biopsy specimens obtained 7 days after starting nCRT are strong predictors of the response to nCRT [5].

Some studies reported that tissue-infiltrating lymphocytes (TILs) are a prognostic factor for colorectal cancer [6] and are related to tumor regression grade (TRG) after nCRT [7]. TILs may vary before and after treatment. We previously reported that the counts of $\mathrm{CD} 3+\mathrm{T}$ cells and FoxP3+ regulatory $\mathrm{T}$ cells increased after chemotherapy with UFT (tegafur uracil) and leucovorin in patients with left-sided colon cancer [8]. In patients with rectal cancer, CD4+, CD8+, PD-L1-positive TILs may vary after nCRT and serve as prognostic factors or histological predictors [9-11]. Prediction of the response to CRT before and soon after treatment may be useful for determining the treatment strategy in individual patients.

We therefore performed H-E staining of TILs using biopsy specimens obtained before nCRT and 7 days after the start of treatment and immunohistochemical staining of lymphocyte surface markers including CD3, CD4, CD8, and FoxP3 to examine the relationship to the histological response, tumor shrinkage rates, and survival rates.

This study was approved by the institutional review board of our university (17R-350), and all patients provided written informed consent.

\section{Patients and Methods}

Our research complies with the guidelines for human studies and was conducted ethically in accordance with the World Medical Association Declaration of Helsinki. Written informed consent was obtained from all patients.

\section{Patients}

The study group comprised 208 patients with clinical T3-T4cN any low-middle rectal adenocarcinomas who received nCRT (40$45 \mathrm{~Gy}$ ) with concurrent oral UFT/leucovorin or S-1 chemotherapy from September 2007 through October 2016.

Histological Evaluation of Biopsy Specimens

Colonoscopy was performed to obtain biopsy specimens before treatment and 7 days (range 4-10 days) after starting preoperative nCRT. We took 6 biopsy specimens from every patient both before and 7 days after the start of nCRT. Biopsy specimens were fixed in formalin, embedded in paraffin, and sliced into $6-\mu \mathrm{m}$-thick serial sections.

\section{Immunohistochemical Staining}

The subtypes of TILs were evaluated by immunohistochemical staining with the surface markers of lymphocytes (CD3, CD4, CD8, and FoxP3), as follows. Formalin-fixed, paraffin-embedded sections were used. Antibodies for the pan T-cell marker CD3 (Ready to use, Clone 2GV6, Roche, Basel, Switzerland), helper Tcell marker CD4 (diluted 1:100, clone 4B12, DAKO, Glostrup, Denmark), cytolytic T-cell marker CD8 (diluted 1:40, C8/144B, DAKO), and regulatory T-cell marker FoxP3 (diluted 1:100, Clone SP97, Thermo Fisher Scientific) were used as primary monoclonal antibodies. An avidin-biotin-peroxidase complex method with diaminobenzidine as chromogen was used according to the manufacturer's instructions. For CD3 and CD8 immunostaining, the Ventana DISCOVERY ULTRA Slide Staining System (Roche Diagnosis) was used. For CD4 and FoxP3 immunostaining, the Leica BOND-MAX fully automatic immunohistochemistry system (Leica Biosystems, Nussloch, Germany) was used. Ventana Cell Conditioning Solution (CCI) was used for $60 \mathrm{~min}$ to perform CD3 and CD8 staining. BOND Epitope Retrieval Solution 2 (AR9640) was used for 20 min to perform CD4 and FoxP3 staining.

\section{Evaluations}

The densities of TILs and lymphocytes were classified into 4 grades (0 to 4 ) according to the results of Black's study [12]: grade 0: no lymphoid infiltrate, grade 1: a few lymphoids scattered, grade 2: a definite scattering of lymphocytes in association with invading cords of tumor cells, grade 3: dense lymphocytic infiltration, with an overall lymphoid appearance of the lesion, grade 4: lymphocytic infiltration intimately related to individual tumor cells, imparting a lymphoid structure even at first glance (Fig. 1a-c).

Grades 0 to 2 were regarded as low TILs and low cell density, and grades 3 to 4 were regarded as high TILs and high cell density. The densities of TILs, CD3+, CD4+, and CD8+ T cells were separately evaluated by 2 physicians (H.M. and S.S.). If different results were obtained, the results were repeatedly evaluated by the 2 physicians using a microscope to determine the grade.

As for FoxP3 regulatory T cells, 4 fields of vision were selected at $\times 400$ magnification. Using Image J (Rasband WS, Image J, US National Institutes of Health, Bethesda, MD, USA), the count of stained lymphocytes was calculated. The cut-off values for FoxP3 in biopsy specimens before nCRT and 7 days after the start of nCRT were calculated using the receiver operating characteristic curve in which a high TRG was regarded as positive (Fig. 1d).

The tumor shrinkage rate was calculated based on the tumor size evaluated on MRI before and after CRT. $\mathrm{T}_{2}$-weighted fast spinecho imaging was performed, and the rate of tumor shrinkage on MRI was calculated by the following formula: tumor shrinkage rate $(\%)=($ tumor volume before CRT - tumor volume before surgery $) /$ tumor volume before CRT $\times 100(\%)$ [13].

Histological regression was classified according to the TRG [14]. TRG was classified as grade 1 (complete regression), grade 2 (presence of rare residual cancer cells), grade 3 (increased number of residual cancer cells), grade 4 (residual cancer outgrowing fibrosis), or grade 5 (absence of regression changes). A TRG of 1 and 2 was defined as marked regression.

\section{Statistical Analysis}

Classification variables were determined by using Fisher's exact test or the $\chi^{2}$ test. Continuous variables were determined by using the Mann-Whitney U test or the Kruskal-Wallis test, and the reproduc- 

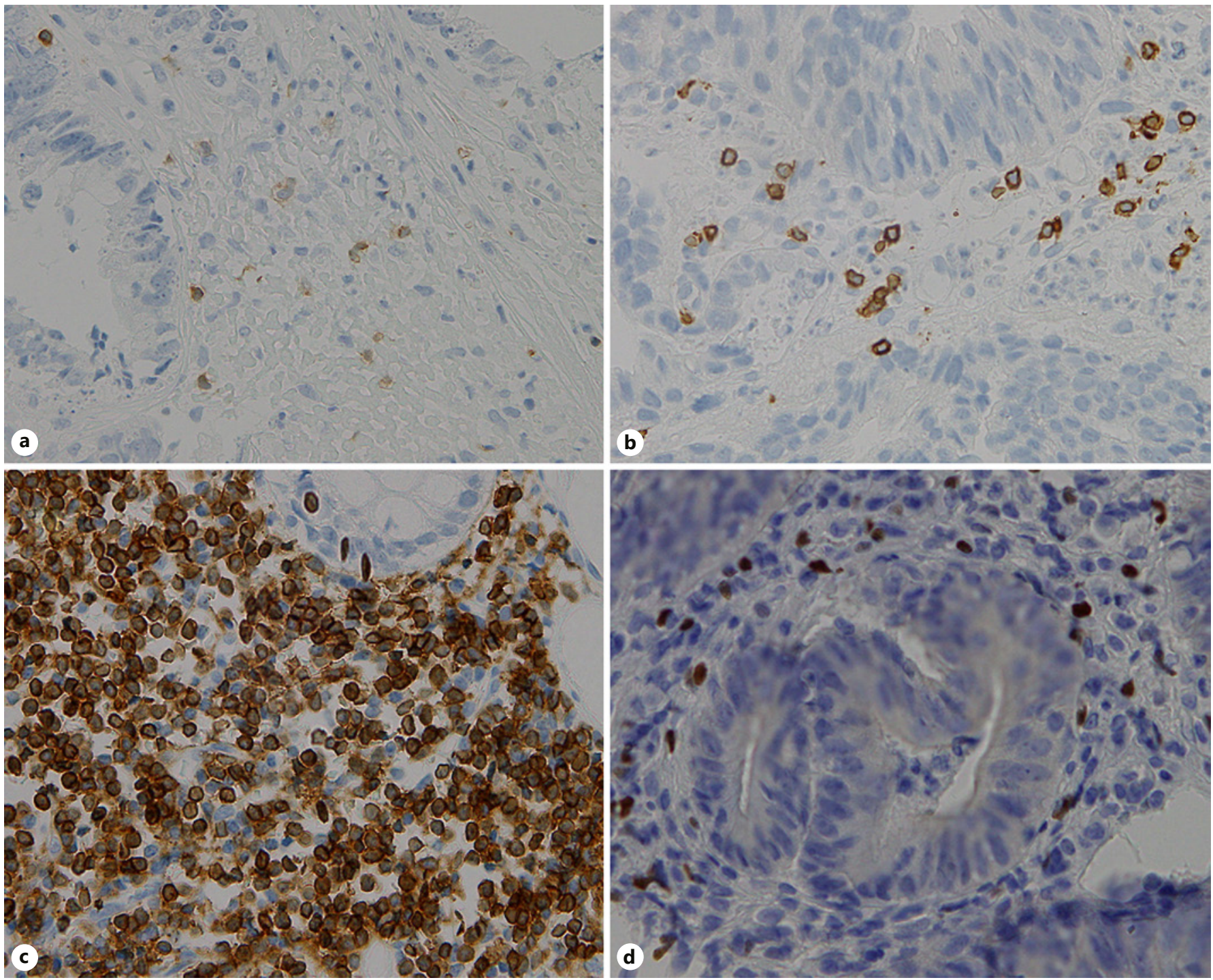

Fig. 1. Examples of stained sections showing different densities of tumor-infiltrating lymphocytes. a Grade 1, a few scattered lymphoids. b Grade 2, a definite scattering of lymphocytes in association with invading cords of tumor cells. c Grade 4, lymphocytic infiltration intimately related to individual tumor cells. d FoxP3 positively stained cells were counted microscopically.

ibility between the 2 groups was determined by Bowker's test. In all analyses, $p$ values of less than 0.05 were considered to indicate statistical significance. The disease-free survival (DFS) rate was calculated by the Kaplan-Meier method. The statistics software package JMP10 (SAS Institute Inc., Cary, NC, USA) was used to analyze the data.

\section{Results}

Table 1 shows the patients' characteristics. Among 208 patients, there were 155 men and 53 women. The mean age was 65 years. Overall, 31 patients (14.9\%) had TRG 1, and 37 patients (17.8\%) had ypStage 0 including pathological complete response.

\section{Comparison of TILs in Biopsy Specimens before}

Treatment and Biopsy Specimens Obtained 7 Days after the Start of $n C R T$

We compared TILs between biopsy specimens obtained before treatment and biopsy specimens obtained 7 days after the start of nCRT (Table 2). FoxP3+ was examined in 170 patients because of a lack of sample size. The values of high TILs on H-E staining and high-den- 
Table 1. Patient characteristics

\begin{tabular}{|c|c|}
\hline \multicolumn{2}{|l|}{ Sex } \\
\hline Male & $155(74.5)$ \\
\hline Female & $53(25.5)$ \\
\hline \multicolumn{2}{|l|}{ Age, years } \\
\hline Median & 65 \\
\hline Mean \pm SD & $64.2 \pm 10$ \\
\hline Range & $33-92$ \\
\hline \multicolumn{2}{|l|}{ Histologic type } \\
\hline wel-mod & $205(98.5)$ \\
\hline por & $3(1.5)$ \\
\hline \multicolumn{2}{|l|}{ Clinical T stage } \\
\hline 2 & $13(6.2)$ \\
\hline 3 & $143(68.8)$ \\
\hline 4 & $52(25.0)$ \\
\hline \multicolumn{2}{|l|}{ Clinical N stage } \\
\hline 0 & $84(40.3)$ \\
\hline 1 & $68(32.7)$ \\
\hline 2 & $39(18.8)$ \\
\hline 3 & $17(8.2)$ \\
\hline \multicolumn{2}{|l|}{ Clinical TNM stage } \\
\hline II & $124(59.6)$ \\
\hline III & $84(40.4)$ \\
\hline \multicolumn{2}{|c|}{ Concurrent chemotherapy } \\
\hline UFT/leucovorin & $56(26.9)$ \\
\hline S1 & $152(73.1)$ \\
\hline \multicolumn{2}{|l|}{ Surgical procedures } \\
\hline LAR & $152(73.1)$ \\
\hline Hartmann & $2(0.9)$ \\
\hline APR & $53(25.5)$ \\
\hline TPE & $1(0.5)$ \\
\hline \multicolumn{2}{|l|}{ TRG } \\
\hline 1 & $31(14.9)$ \\
\hline 2 & $56(26.9)$ \\
\hline 3 & $79(38.0)$ \\
\hline 4 & $42(20.2)$ \\
\hline \multicolumn{2}{|l|}{ ypStage } \\
\hline 0 & $37(17.8)$ \\
\hline I & $56(26.9)$ \\
\hline II & $67(32.2)$ \\
\hline III & $48(23.1)$ \\
\hline
\end{tabular}

Values are given as $n(\%)$. wel, well-differentiated tubular adenocarcinoma; mod, moderately differentiated tubular adenocarcinoma; por, poorly differentiated adenocarcinoma; LAR, low anterior resection; APR, abdominoperineal resection; TPE, total pelvic exenteration; TRG, tumor regression grade.

sity CD3+, CD4+, CD8+, and FoxP3+ T cells on immunostaining were $67,36,26,6$, and $58 \%$, respectively. In biopsy specimens obtained 7 days after the start of nCRT, the value of high TILs on $\mathrm{H}$-E staining was $64.9 \%$, which did not differ significantly from that before CRT. However, the values of high-density $\mathrm{CD} 3+, \mathrm{CD} 4+$, $\mathrm{CD} 8+$, and FoxP3+ $\mathrm{T}$ cells on immunostaining were
Table 2. Comparison of TILs in biopsy specimens before treatment and biopsy specimens obtained 7 days after the start of nCRT

\begin{tabular}{lccc}
\hline & $\begin{array}{l}\text { Before } \\
\text { CRT }\end{array}$ & $\begin{array}{l}\text { After starting } \\
\text { CRT }\end{array}$ & $p$ value \\
& & & \\
Hematoxylin and eosin staining & & \\
$\quad$ High TILs & $139(67)$ & $135(65)$ & $0.6115^{*}$ \\
Low TILs & $69(33)$ & $73(35)$ & \\
CD3+ & & & \\
High & $74(36)$ & $25(12)$ & $<0.0001^{*}$ \\
Low & $134(64)$ & $183(88)$ & \\
CD4+ & & & \\
High & $53(26)$ & $21(10)$ & $<0.0001^{*}$ \\
Low & $155(75)$ & $187(90)$ & \\
CD8+ & & & \\
High & $12(6)$ & $1(1)$ & $0.0023^{*}$ \\
Low & $196(94)$ & $207(99)$ & \\
FoxP3 & & & \\
Mean \pm SD & $211 \pm 98.6$ & $134 \pm 71.9$ & $<0.0001^{\dagger}$ \\
High & $99(58)$ & $78(46)$ & $0.0046^{*}$ \\
Low & $71(42)$ & $92(54)$ & \\
\hline
\end{tabular}

* Bowker test. ${ }^{\dagger}$ Student’s $t$ test

$12.0,10.0,0.5$, and $45.9 \%$, respectively, which were significantly decreased, as compared with those before CRT $(p<0.0001, p<0.0001, p=0.0023$, and $p=0.0046$, respectively).

\section{Relations of the Cell Densities of TILs and}

Lymphocytes in Biopsy Specimens Obtained before $n C R T$ to Histological Response, Tumor Shrinkage

Rates, and the 3-Year DFS Rate (Table 3)

In biopsy specimens obtained before $\mathrm{nCRT}$, the rate of marked histological regression (TRG 1 or 2) was significantly higher in patients with high-density FoxP3 cells (51\%) than in those with low-density FoxP3 cells (35\%, $p=0.0342)$. No factors were related to the rate of TRG 1 . The tumor shrinkage rate was significantly higher in patients with high-density CD4+ and FoxP3+ cells (79.0 and $75.7 \%$, respectively) than in those with low-density CD4+ and FoxP3+ cells $(p=0.0052$ and $p=0.033)$. No factors were related to the 3 -year DFS rate.

\section{Relations of the Cell Densities of TILs and} Lymphocytes in Biopsy Specimens 7 Days after the Start of $n C R T$ to the Histological Response, Tumor Shrinkage Rate, and the 3-Year DFS rate (Table 4)

In biopsy specimens obtained 7 days after the start of nCRT, the marked histological regression rate was significantly higher in patients who had high-density CD4+ 
Table 3. Relations of the cell densities of TILs and lymphocytes in biopsy specimens obtained before nCRT to histological response, tumor shrinkage rates, and the 3 -year disease-free survival rate

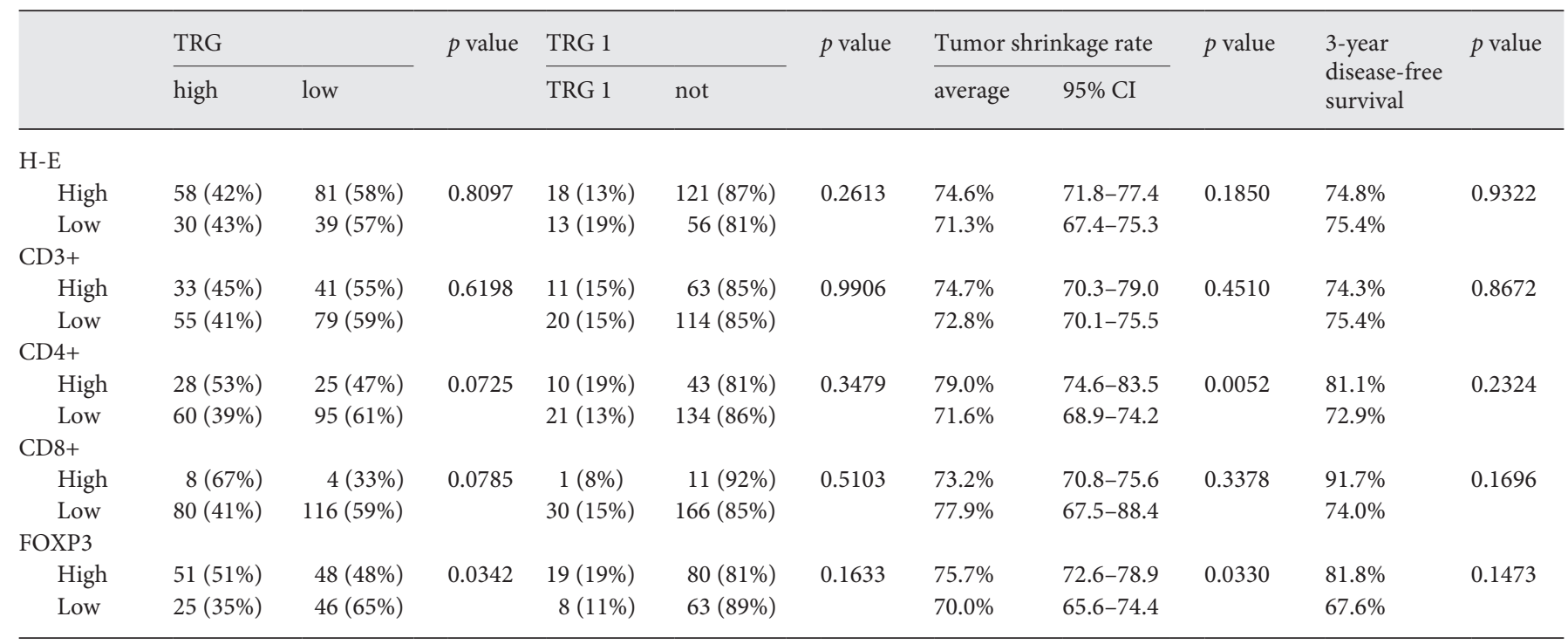

Table 4. Relations of the cell densities of TILs and lymphocytes in biopsy specimens 7 days after the start of nCRT to the histological response, tumor shrinkage rate, and the 3 -year DFS rate

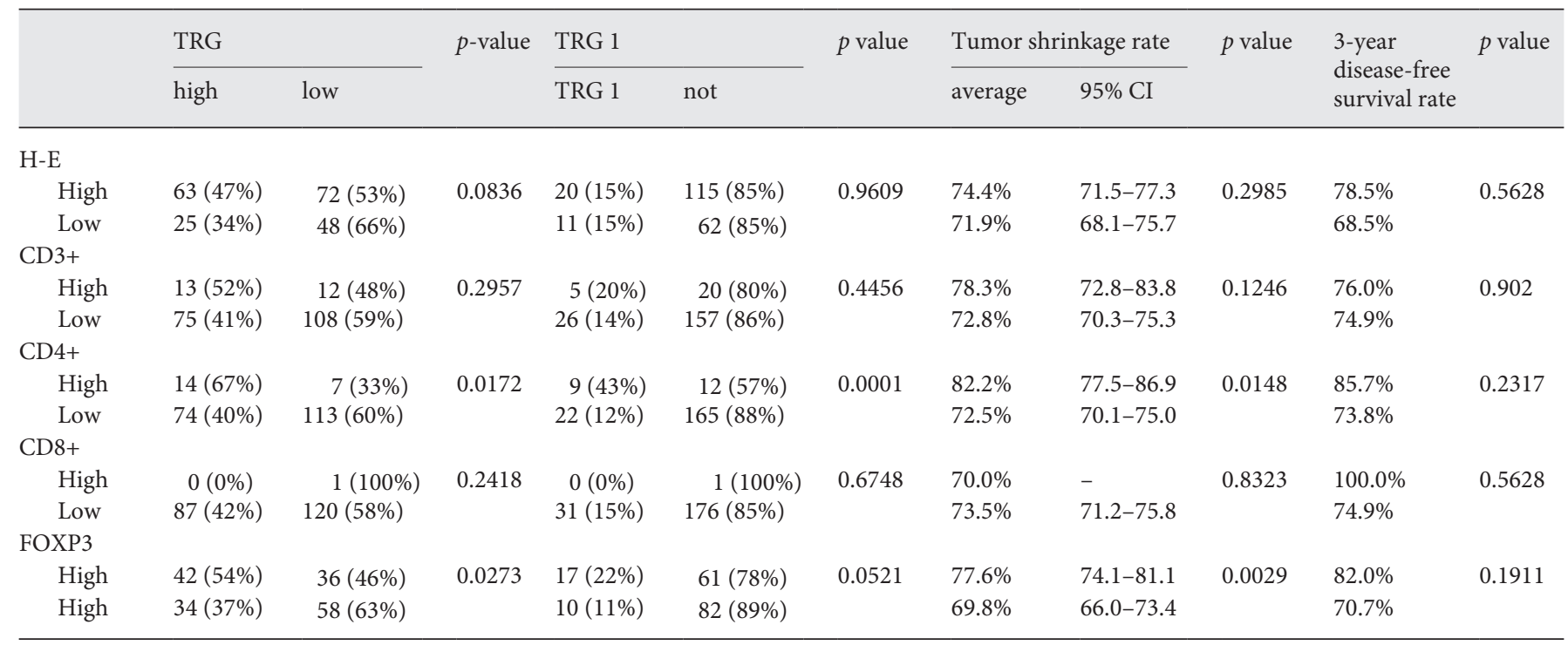

and FoxP3 cells (67 and 54\%, respectively) than in those who had low-density CD $4+$ and FoxP3 cells $(p=0.0172$ and $p=0.0273$ ). The percentage of TRG 1 was significantly higher in patients with high-density CD4+ T cells (43\%) than in those with low-density CD4+ T cells (12\%) $(p=0.0001)$. The tumor shrinkage rates were
$82.2 \%$ in patients with high-density CD4+ cells and $77.6 \%$ in patients with high-density FoxP3 cells, which were significantly higher than the respective rates in patients with low-density CD4+ and FoxP3 cells $(p=$ 0.0148 and $p=0.0029$ ). No factors were related to the 3-year DFS rate. 


\section{Discussion}

In patients with colorectal cancer, inflammatory-cell infiltration has been reported to be associated with survival rates, irrespective of pathological stage [15]. Tumorassociated macrophages, dendritic cells, and tumor-infiltrating T lymphocytes are considered a symbol of the immune response [15]. As for subtypes of $\mathrm{T}$ cells, surface markers including CD3+, CD4+, CD8+, and FoxP3+ T cells have been reported to be associated with histological response to nCRT or survival [16-19].

Yasuda et al. [20] reported that patients with highdensity CD4+ and CD8+ T cells in biopsy specimens who received nCRT for rectal cancer had high rates of tumor shrinkage after nCRT and pathological complete response. Our results regarding the value of CD4+ T cell density were consistent with those of their study. However, no relation was observed between the value of CD8+ $\mathrm{T}$ cell density and response to nCRT.

TILs have been reported to vary depending on treatment $[8,9]$. In the present study, to clarify the relation between changes in $\mathrm{T}$ cells soon after the start of treatment and histological response, we compared biopsy specimens before and after nCRT. In our study, the values of high-density CD3+, CD4+, CD8+, and FoxP3+ T cells in biopsy specimens obtained 7 days after the start of nCRT were significantly lower than the respective values in biopsy specimens obtained before nCRT. However, Teng et al. [16] reported that the value of CD4+ T cell density was significantly higher in resected specimens after CRT than in biopsy specimens before treatment. We evaluated biopsy specimens soon after the start of nCRT. Teng et al. [16] evaluated resected specimens at the time of surgery 2-3 weeks after nCRT. The value of CD4+ T cell density may have been affected by a difference in evaluation time.

Regulatory T cells expressing Foxp 3 may inhibit the activation of T cells. Some high-density FoxP3+ tumors have been reported to have poor outcomes $[21,22]$. In patients with colorectal cancer, some studies reported that high-density FoxP3+ cells have poor outcomes [23]. Other studies reported that high-density FoxP3+ cells have good outcomes [24]. This point remains controversial. In our study, no significant relation was observed between DFS and FOXP3+ T cells in biopsy specimens obtained before nCRT or 7 days after the start of nCRT.

In our study, patients with high-density FoxP3 + T cells in biopsy specimens obtained before nCRT and 7 days after starting nCRT were associated with a good histological response. These results may support the previous report that high-density TIL tumors are originally associated with a high immunogenicity and promote the release of tumorspecific antigens, leading to a good response to nCRT [16].

Our results suggested that $\mathrm{T}$ cell-mediated immune response plays an important role in the tumor response to nCRT. Particularly, CD4+ and FOXP3+ TIL densities were demonstrated to be related to histological response to nCRT. In addition, we found that biopsy specimens obtained 7 days after treatment are more useful for predicting response to nCRT than biopsy specimens obtained before treatment.

\section{Conclusions}

In patients who received nCRT for rectal cancer, the results of immunostaining for CD4+ and FOXP3+ TILs in biopsy specimens obtained 7 days after treatment may be more closely related to the histological response to nCRT and tumor shrinkage rates than the respective values in biopsy specimens obtained before nCRT.

\section{Statement of Ethics}

This study was approved by the institutional review board of Tokai University (17R-350). Our research complies with the guidelines for human studies and was conducted ethically in accordance with the World Medical Association Declaration of Helsinki. Written informed consent was obtained from all patients.

\section{Conflict of Interest Statement}

Hiroshi Miyakita, Sotaro Sadahiro, Toshiyuki Suzuki, Lin Fung Chan, Takashi Ogimi, Kazutake Okada, Seiichiro Yamamoto, and Hiroshi Kajiwara have no potential conflicts of interest to declare.

\section{Funding Sources}

There was no funding source for this study.

\section{Author Contributions}

Conception and design: H. Miyakita, S. Sadahiro, T. Suzuki. Provision of study materials or patients: H. Miyakita, L.F. Chan, T. Ogimi, K. Okada, T. Suzuki. Collection and assembly of data: H. Miyakita, T. Suzuki, K. Okada, S. Sadahiro. Data analysis and interpretation: H. Miyakita, S. Sadahiro, H. Kajiwara. Manuscript writing: H. Miyakita, S. Sadahiro. Final approval of manuscript: H. Miyakita, S. Sadahiro, T. Suzuki, L.F. Chan, T. Ogimi, K. Okada, S. Yamamoto, and H. Kajiwara. 


\section{References}

1 Peeters KC, Marijnen CA, Nagtegaal ID, Kranenbarg EK, Putter $\mathrm{H}$, Wiggers $\mathrm{T}$, et al. The TME trial after a median follow-up of 6 years: increased local control but no survival benefit in irradiated patients with resectable rectal carcinoma. Ann Surg. 2007;246(5): 693-701.

2 Sauer R, Becker H, Hohenberger W, Rödel C, Wittekind C, Fietkau R, et al. Preoperative versus postoperative chemoradiotherapy for rectal cancer. N Engl J Med. 2006;351(17): 1731-40.

3 Gérard JP, Conroy T, Bonnetain F, Bouché O, Chapet O, Closon-Dejardin MT, et al. Preoperative radiotherapy with or without concurrent fluorouracil and leucovorin in T3-4 rectal cancers: results of FFCD 9203. J Clin Oncol. 2006;24(28):4620-5.

4 Suzuki T, Sadahiro S, Tanaka A, Okada K, Saito G, Miyakita H, et al. A Modified Classification of Prognostic Factors Based on Pathological Stage and Tumor Regression Grade in Patients with Rectal Cancer Who Receive Preoperative Chemoradiotherapy. Oncology. 2017;93(5):287-94.

5 Suzuki T, Sadahiro S, Tanaka A, Okada K, Kamata $\mathrm{H}$, Kamijo A, et al. Biopsy specimens obtained 7 days after starting chemoradiotherapy (CRT) provide reliable predictors of response to CRT for rectal cancer. Int J Radiat Oncol Biol Phys. 2013;85(5):1232-8.

6 Galon J, Costes A, Sanchez-Cabo F, Kirilovsky A, Mlecnik B, Lagorce-Pagès C, et al. Type, density, and location of immune cells within human colorectal tumors predict clinical outcome. Science. 2006;313(5795):1960-4.

7 Anitei MG, Zeitoun G, Mlecnik B, Marliot F, Haicheur N, Todosi AM, et al. Prognostic and predictive values of the immunoscore in patients with rectal cancer. Clin Cancer Res. 2014;20(7):1891-9.

8 Sadahiro S, Suzuki T, Tanaka A, Okada K, Saito G, Miyakita H, et al. Induction of CD3+ and FoxP3 + T cells in left-sided colorectal tumors after UFT/LV chemotherapy. Anticancer Res. 2019;39(4):1997-2005.
9 Yu JL, Jaemoon K, Sehui K, Jeon SR, Chie EK, Kim K, et al. Chemoradiation-induced alteration of programmed death-ligand 1 and CD8+ tumor-infiltrating lymphocytes identified patients with poor prognosis in rectal cancer: a matched comparison analysis. Int J Radiat Oncol Biol Phys. 2017;99:2216-1224.

10 Kamran SC, Lennerz JK, Margolis CA, Liu D, Reardon B, Wankowicz SA, et al. Integrative molecular characterization of resistance to neoadjuvant chemoradiation in rectal cancer. Clin Cancer Res. 2019;25(18):5561-71.

11 Matsutani S, Shibutani M, Maeda K, Nagahara H, Fukuoka T, Nakao S, et al. Significance of tumor-infiltrating lymphocytes before and after neoadjuvant therapy for rectal cancer. Cancer Sci. 2018;109(4):966-79.

12 Black MM, Speer FD, Opler SR. Structural representations of tumor-host relationships in mammary carcinoma; biologic and prognostic significance. Am J Clin Pathol. 1956; 26(3):250-65.

13 Suzuki T, Sadahiro S, Tanaka A, Okada K, Saito G, Kamijo A, et al. Relationship between histologic response and the degree of tumor shrinkage after chemoradiotherapy in patients with locally advanced rectal cancer. J Surg Oncol. 2014;109(7):659-64.

14 Mandard AM, Dalibard F, Mandard JC, Marnay J, Henry-Amar M, Petiot JF, et al. Pathologic assessment of tumor regression after preoperative chemoradiotherapy of esophageal carcinoma. Clinicopathologic correlations. Cancer. 1994;73(11):2680-6.

15 Roxburgh CS, McMillan DC. The role of the in situ local inflammatory response in predicting recurrence and survival in patients with primary operable colorectal cancer. Cancer Treat Rev. 2012;38(5):451-66.

16 Teng F, Meng X, Kong L, Mu D, Zhu H, Liu $S$, et al. Tumor-infiltrating lymphocytes, forkhead box P3, programmed death ligand-1, and cytotoxic T lymphocyte-associated antigen-4 expressions before and after neoadjuvant chemoradiation in rectal cancer. Transl Res. 2015;166(6):721-732.e1.
17 Teng F, Mu D, Meng X, Kong L, Zhu H, Liu $S$, et al. Tumor infiltrating lymphocytes (TILs) before and after neoadjuvant chemoradiotherapy and its clinical utility for rectal cancer. Am J Cancer Res. 2015;5(6):2064-74.

18 Shinto E, Hase K, Hashiguchi Y, Sekizawa A, Ueno H, Shikina A, et al. CD8+ and FoxP3+ tumor-infiltrating $\mathrm{T}$ cells before and after chemoradiotherapy for rectal cancer. Ann Surg Oncol. 2014;21(Suppl 3):S414-21.

19 Noble F, Mellows T, McCormick Matthews LH, Bateman AC, Harris S, Underwood TJ, et al. Tumour infiltrating lymphocytes correlate with improved survival in patients with oesophageal adenocarcinoma. Cancer Immunol Immunother. 2016;65(6):651-62.

20 Yasuda K, Nirei T, Sunami E, Nagawa H, Kitayama J. Density of CD4(+) and CD8(+) T lymphocytes in biopsy samples can be a predictor of pathological response to chemoradiotherapy (CRT) for rectal cancer. Radiat Oncol. 2011;6:49-54.

21 Curiel TJ, Coukos G, Zou L, Alvarez X, Cheng $\mathrm{P}$, Mottram P, et al. Specific recruitment of regulatory $\mathrm{T}$ cells in ovarian carcinoma fosters immune privilege and predicts reduced survival. Nat Med. 2004;10(9):942-9.

22 deLeeuw RJ, Kost SE, Kakal JA, Nelson BH. The prognostic value of FoxP3+ tumor-infiltrating lymphocytes in cancer: a critical review of the literature. Clin Cancer Res. 2012; 18(11):3022-9.

23 Saito T, Nishikawa H, Wada H, Nagano Y, Sugiyama D, Atarashi K, et al. Two FOXP3(+) CD4(+) T cell subpopulations distinctly control the prognosis of colorectal cancers. Nat med. 2016;22(6):679-84.

24 Salama P, Phillips M, Grieu F, Morris M, Zeps $\mathrm{N}$, Joseph D, et al. Tumor-infiltrating FoxP3+ $T$ regulatory cells show strong prognostic significance in colorectal cancer. J Clin Oncol. 2009;27(2):186-92.
Tissue-Infiltrating Lymphocytes as Predictive Factor of nCRT
Oncology 2020;98:869-875

DOI: $10.1159 / 000508922$ 